\title{
Maturação das vértebras cervicais e sua correlação com a idade óssea da mão e punho como indicadores no tratamento ortodôntico*
}

\author{
Vitoria Aparecida Muglia Moscatiello**, Henrique Lederman***,

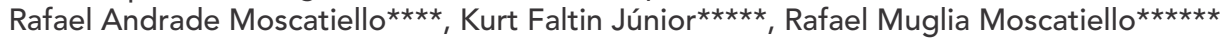

\begin{abstract}
Resumo
Objetivos: a proposta deste estudo prospectivo, transversal e multicêntrico foi correlacionar as alterações morfológicas das vértebras cervicais $\mathrm{C} 2, \mathrm{C} 3$ e C4, com a idade cronológica e comparar com a idade esqueletal dos ossos da mão e punho; e verificar se existem diferenças no padrão de crescimento ósseo entre os gêneros masculino e feminino. Metodologia: a amostra foi constituída por 140 pacientes, 74 do gênero feminino e 66 do masculino. A avaliação das alterações das vértebras cervicais foi feita pelo método de Hassel e Farman modificado por Baccetti, Franchi e McNamara para 5 estágios que se correlacionam com o crescimento puberal em telerradiografias cranianas em norma lateral. As radiografias da mão e punho avaliaram os eventos de ossificação por meio do método de Greulich e Pyle. Resultados: os resultados demonstraram correlação dos estágios de maturação das vértebras cervicais com a idade cronológica; e correlação positiva e moderada, estatisticamente significante entre os dois métodos com $r=0,6326$ (IC $=95 \%)$ e $p<0,0001$. Conclusões: desta forma, pôde-se concluir que a idade aumenta proporcionalmente em relação aos estágios de maturação, sendo que a maioria dos pacientes da amostra encontrou-se no estágio II, considerado bom para o início da terapêutica ortodôntico/ortopédica. O nível de correlação entre os métodos demonstrou que a avaliação dos estágios de maturação das vértebras cervicais é um método adicional útil e confiável na determinação do estágio de crescimento facial nas crianças em crescimento puberal. $\mathrm{O}$ gênero feminino apresentou estágios de crescimento mais precoces que $\mathrm{o}$ gênero masculino, atingindo o início e o final do surto de crescimento puberal em idade cronológica mais inferior.
\end{abstract}

Palavras-chave: Maturação óssea. Crescimento puberal. Maturação da mão e do punho. Vértebras cervicais.

* Artigo referente à Tese de Doutorado de Vitoria Aparecida Muglia Moscatiello junto ao Departamento de Diagnóstico por Imagem - Escola Paulista de Medicina - Universidade Federal de São Paulo - UNIFESP.

** Doutora pelo Departamento de Diagnóstico por Imagem da UNIFESP/EPM. Professora de Ortodontia da Universidade Paulista - UNIP.

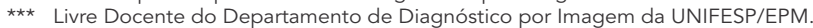

Doutor pelo Departamento de Diagnóstico por Imagem da UNIFESP/EPM. Professor do Curso de Especialização em Implantodontia da Universidade Paulista - UNIP.

Chefe do Departamento de Ortodontia e Coordenador do Curso de Especialização em Ortodontia da Universidade Paulista - UNIP.

$\begin{array}{ll}\star \star \star \star \star \star & \text { Chefe do Departamento de Ortodontia e Coordenador do Curso } \\ \star \star \star \star \star \star & \text { Cirurgião-dentista graduado pela Universidade Paulista - UNIP. }\end{array}$ 


\section{INTRODUÇÃO}

As informações que o estudo do crescimento e do desenvolvimento craniofacial proporciona têm fundamental importância para se estabelecer a possivel etiologia das desordens craniofaciais, o diagnóstico individual preciso e um planejamento de tratamento adequado. É importante, para o tratamento médico e odontológico, estimar quanto uma criança progride em relação à maturidade, observando-se que as variações individuais da velocidade em que ela se transforma em adulto dependem das influências genéticas, ambientais e nutricionais, entre outras ${ }^{29}$.

Do nascimento à idade adulta, as alterações são morfológicas, fisiológicas e psíquicas, intensificadas, principalmente, antes e durante a puberdade ${ }^{30}$. Enquanto na infância o crescimento relaciona-se intimamente com a idade cronológica; conforme a puberdade se aproxima, esta relação não se estabelece $^{27}$. O que se observa é uma ocorrência dos eventos de crescimento, numa seqüência razoavelmente constante, onde a idade cronológica em que eles terminam varia muito, não coincidindo com a idade de maturação biológica ${ }^{24}$.

O grau de maturação alcançado por um indivíduo pode ser avaliado por vários indicadores como: (a) a idade morfológica, avaliada por meio das medidas das diferentes partes do corpo; (b) a idade de maturação óssea, por meio da avaliação do avanço do desenvolvimento dos ossos da mão e punho, que possuem um grande número de ossos e epífises em desenvolvimento; (c) a idade de maturação sexual, por meio das mudanças que ocorrem na adolescência; (d) a idade de maturação dentária, por meio da formação e erupção dos dentes ${ }^{19}$.

A idade óssea tem sido o indicador mais comumente usado nos estudos sobre crescimento e desenvolvimento e é considerada um verdadeiro registro da idade biológica. Sua determinação está baseada nas mudanças esqueléticas que podem ser visualizadas em radiografias de áreas selecionadas do corpo, durante todo o período de crescimento ${ }^{28}$.

O método de avaliação da maturação esquele- tal comumente usado, desde 1959, é o de Greulich e Pyle, que utiliza radiografias de mão e punho e realiza leituras sucessivas do desenvolvimento da criança ou de seu progresso ${ }^{15}$.

Entre os métodos de avaliação da idade biológica, muitas pesquisas têm sido desenvolvidas a partir dos achados de Lamparski ${ }^{17}$, o primeiro autor que - observando as mudanças no tamanho e na forma das vértebras cervicais em telerradiografias cranianas em norma lateral - descreveu seis estágios de maturação, mais precisamente da segunda à sexta vértebra. Assim confirmou, pelo resultado estatístico, ser este método válido e confiável, apresentando o mesmo valor clínico que o método de Greulich e Pyle de avaliação pelas modificações da mão e do punho' ${ }^{17}$.

Hassel e Farman ${ }^{16}$, baseando-se nas observações de Lamparski ${ }^{17}$, desenvolveram o IMVC (Índice de Maturação das Vértebras Cervicais) avaliando as alterações morfológicas das vértebras cervicais C2, C3 e C4, e determinando o surto de crescimento puberal remanescente.

A efetividade deste método para determinar o melhor período de aplicação de tratamentos ortopédicos foi avaliada em outros estudos, como os de Baccetti et al. ${ }^{1}$ Os achados de Franchi et al. ${ }^{11}$ comprovaram que ele é um método apropriado de avaliação da maturação esqueletal dos pacientes. Em 2002, Baccetti et al., ${ }^{2,3}$ apresentaram uma nova versão do método EMVC (Estágio de Maturação das Vértebras Cervicais), reduzindo de seis para cinco estágios de avaliação.

Em Ortodontia, a determinação da maturação óssea é um fator de grande importância no planejamento dos tratamentos, principalmente dos ortopédicos, porque estabelece a melhor época do seu início, aproveitando o próprio crescimento do paciente para as correções dos problemas transversos, verticais e, principalmente, dos ântero-posteriores, e correção das alterações ósseas e neuromusculares, procurando a normalização do sistema mastigatório e, conseqüentemente, a harmonia facial ${ }^{9}$. 
A variedade de métodos para a determinação da idade óssea, pelo interesse em se identificar a ocorrência dos surtos de crescimento, e a aplicabilidade do método de avaliação pelas vértebras cervicais despertaram grande interesse para esta investigação científica, que propôs-se a:

1) correlacionar as alterações morfológicas das vértebras cervicais $\mathrm{C} 2$, C3 e C4 com a idade cronológica e comparar com a idade esqueletal dos ossos da mão e do punho;

2) verificar se existem diferenças no padrão de crescimento ósseo entre os gêneros masculino e feminino.

\section{MATERIAL E MÉTODOS}

Este estudo transversal, prospectivo e multicêntrico utilizou, como amostra, pacientes da Clínica Infantil da Universidade Paulista (UNIP), que foram selecionados pelo Setor Triagem e encaminhados para tratamento odontopediátrico e ortodôntico interceptativo.

A amostra foi constituída de 140 crianças de etnia variada, com idade entre 42 e 164 meses, nas quais foram realizadas e avaliadas 140 telerradiografias cranianas em norma lateral e radiografias de mão e punho. Para a obtenção das telerradiografias cranianas em norma lateral, a posição da cabeça seguiu o critério de manter o plano de Frankfurt paralelo ao solo e horizontal ao plano do filme ${ }^{18,23}$. A distância foco/filme de 1,56 metros foi fixada no aparelho e manteve-se sempre a mesma. As radiografias de mão e punho foram feitas com a mão espalmada, procurando-se obter um maior contato do filme com o eixo do dedo médio em linha reta com o eixo do antebraço; os dedos e o polegar posicionaram-se em um ângulo de, mais ou menos, $30^{\circ} \mathrm{com}$ o dedo indicador.

A idade óssea por meio da radiografia de mão e punho foi avaliada determinando-se a maturação óssea pelo grau de mineralização dos ossos das epífises, dos metacarpianos e das falanges e dos ossos do punho (Fig. 1). A interpretação destas radiografias foi realizada no Departamento de Diag-

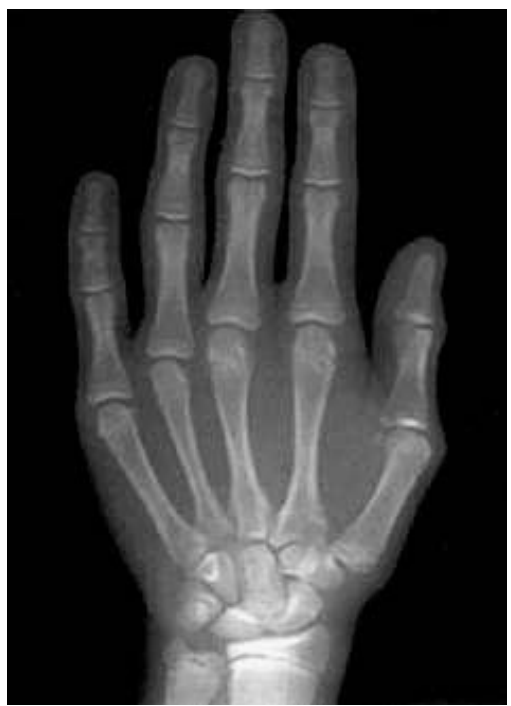

FIGURA 1 - Radiografia da mão e punho de um paciente do gênero feminino com 10 anos de idade: ossos carpais, metacarpianos e falanges.

nóstico por Imagem, sendo comparadas com um Atlas de Greulich e Pyle ${ }^{15}$. Foi utilizada a tabela de desvio-padrão determinada pelos estudos da Brush Foundation, e também as tabelas de acordo com a padronização dos intervalos de padrão cronológico, tanto para o gênero masculino, quanto para o feminino.

Nas telerradiografias cranianas em norma lateral foram avaliados os estágios das CVM pelo método visual e também foram confeccionados traçados do contorno anatômico da segunda (C2), terceira (C3) e quarta (C4) vértebras cervicais. As variações morfológicas visíveis para determinar os referidos estágios são: presença de concavidades nas bordas inferiores das vértebras cervicais $\mathrm{C} 2$, $\mathrm{C} 3$ e C4; forma das vértebras $\mathrm{C} 3$ e $\mathrm{C} 4$ em quatro alterações morfológicas, na seqüência de desenvolvimento (Fig. 2).

O método utilizado para avaliação da maturação das vértebras cervicais foi o de Hassel e Farman modificado por Baccetti, Franchi e Mc$\mathrm{Namara}^{2}$ em 5 estágios que têm correlação com o crescimento puberal (Fig. 3):

- CVMS I: bordas inferiores da C2, C3 e C4 


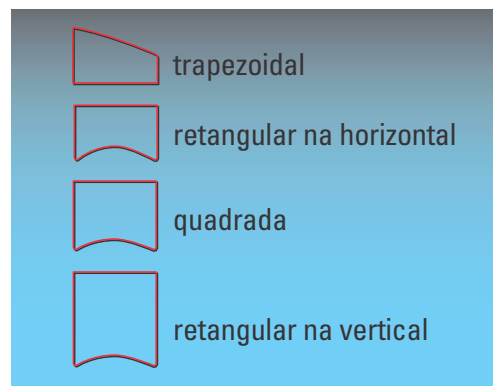

FIGURA 2 - Esquema das formas das vértebras cervicais e as quatro alterações morfológicas.

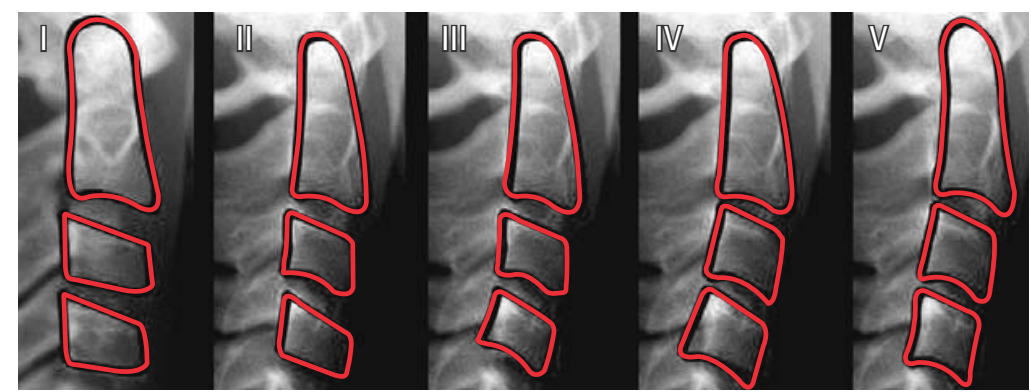

FIGURA 3 - Os cinco estágios de avaliação das CVM e sua correlação com o crescimento puberal. planas ou achatadas, com possibilidade de existir uma leve concavidade na C2; forma trapezoidal da C3 e C4, afuniladas de posterior para anterior. $\mathrm{O}$ pico de crescimento não ocorre antes de um ano após este estágio.

- CVMS II: presença de concavidades distintas nas bordas inferiores da C2 e C3; C3 e C4 apresentam-se trapezoidais ou retangulares na horizontal. O pico de crescimento ocorre neste estágio.

- CVMS III: presença de concavidades distintas nas bordas inferiores da C2, C3 e C4; C3 e C4 apresentam-se retangulares na horizontal. $\mathrm{O}$ pico de crescimento termina neste estágio ou ocorreu durante o ano antes deste estágio.

- CVMS IV: presença de concavidades acentuadas nas bordas inferiores da C2, C3 e C4; C3 e $\mathrm{C} 4$ apresentam-se quadradas. $\mathrm{O}$ pico de crescimento ocorreu um ano antes deste estágio.

- CVMS V: presença de concavidades profundas nas bordas inferiores da C2, C3 e C4; C3 e C4 apresentam-se retangulares na vertical. $\mathrm{O}$ pico de crescimento ocorreu pelo menos dois anos antes deste estágio.

\section{Avaliação estatística}

Após a obtenção das medidas necessárias para o desenvolvimento do estudo proposto, as variáveis numéricas foram submetidas aos testes de normalidade de Shapiro-Wilk e Kolmorov-Smirnov, sendo expressas em valores médios e desvios-padrão, ou em mediana e percentis. A análise de variância de um fator foi realizada com a finalidade de se verificar, por meio do teste $t$ de Bonferroni e pelo teste de Kruskal-Wallis, a existência de diferenças significativas entre os grupos estudados.

A existência de correlação entre os valores das idades cronológica e óssea e os CVMS foi pesquisada pelo cálculo do coeficiente de correlação de Spearman (r). A força de correlação referiu-se ao grau de proximidade do coeficiente $r$ das variáveis, que pode estar dentro do intervalo de $-1 \mathrm{a}+1$, onde quanto mais próximo a +1 maior a correlação positiva, e quanto mais próxima de -1 maior a correlação inversa.

Foi considerado o nível de significância de 5\%, sendo que as variáveis foram descritas por médias e intervalos de confiança (IC) de 95\% (quando a verdadeira média da população está dentro deste intervalo). Para a distribuição dos valores foi utilizado um boxplot. Com ele, pode-se ter idéia de como os dados se distribuem.

\section{RESULTADOS}

A amostra contendo o número de 140 crianças de etnia variada foi constituída de 66 pacientes do gênero masculino (47\%) e 74 do gênero feminino (53\%).

A média de idade dos pacientes da amostra foi de 109 meses, variando entre 42 e 164 meses. No gráfico 1 observa-se a distribuição do número de pacientes de acordo com o gênero e a idade cronológica. 


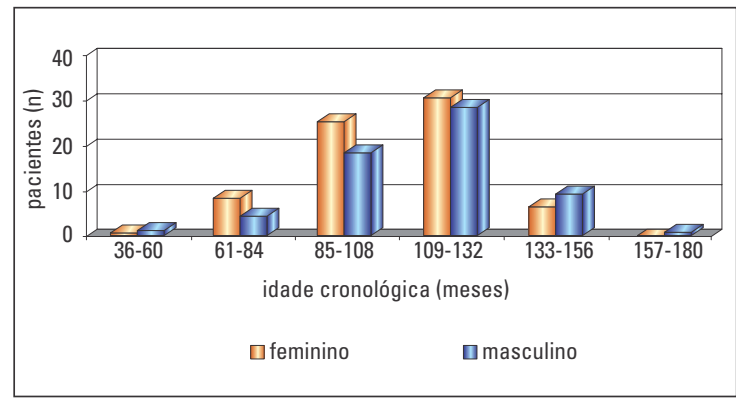

GRÁFICO 1 - Distribuição do número de pacientes por gênero e idade cronológica.

\section{Correlação das alterações morfológicas das vértebras cervicais $\mathrm{C} 2, \mathrm{C} 3$ e C4 com a idade cronológica}

Observou-se que 24 pacientes encontraram-se no estágio I, 94 no estágio II, 20 no estágio III e 2 no estágio IV. A avaliação da correlação da idade cronológica com os EMVC foi observada pelo cálculo da média e da mediana, sendo que os valores utilizados nos cálculos estatísticos estão apresentados na tabela 1 .

Pela análise descritiva dos dados da tabela 1 foram encontrados os valores que representam $o$ mínimo e o máximo das idades dos pacientes para cada um dos EMVC. Pode-se observar que a idade média foi de 87,67 meses no estágio I; de 110,4 meses no estágio II; 130,5 meses no estágio III e 143,5 meses no estágio IV.

$\mathrm{Na}$ correlação entre os valores da idade cronológica e os EMVC, em todos os pacientes foi observado or $=0,5830(\mathrm{IC}=95 \% ; 0,4578$ a 0,6855$)$, com $\mathrm{p}<0,0001$.
Com o uso do boxplot, pode-se ter idéia de como os dados se distribuem. Para construí-lo, os valores foram divididos em quatro grupos (quartis), com os valores da tabela 1 usados no gráfico 2 .

$\mathrm{Na}$ amostra havia 24 pacientes no estágio I de MVC, 19 apresentando-se na faixa etária entre 61e 84 meses e de 85 a 108 meses de idade. A grande maioria dos pacientes encontra-se no estágio II de MVC, com maior prevalência na faixa etária entre 85 e 132 meses (Gráf. 3).

\section{Correlação das alterações morfológicas das vértebras cervicais $\mathrm{C2}$, C3 e C4 com a matura- ção dos ossos da mão e punho}

A distribuição da correlação da idade óssea estabelecida pelo método de Greulich e Pyle com os EMVC foi observada pelo cálculo da mediana, sendo o valor de $r=0,6326$ (IC 95\%, 0,5177 a 0,7250 ), com $p<0,0001$, estando apresentados no gráfico 4 .

\section{Variação dos estágios de maturação das vér- tebras cervicais C2, C3 e C4 entre os gêneros masculino e feminino}

Quando os pacientes foram separados em dois grupos (de acordo com o gênero), observou-se para a correlação entre os valores da idade cronológica e os EMVC - os valores do coeficiente de correlação de Spearman, conforme a tabela 2.

Estes valores demonstraram que o gênero feminino, tanto na idade cronológica quanto nos EMVC, encontrou-se mais adiantado que o masculino, com uma correlação considerada entre positiva média e positiva forte.

Tabela 1 - Idade cronológica em meses e os EMVC.

\begin{tabular}{cccccc}
\hline EMVC & $\mathbf{n}$ & média \pm desvio-padrão & mínimo (máximo) & mediana & $\mathbf{2 5 \%}(\mathbf{7 5 \%})$ \\
\hline I & 24 & $87,67 \pm 17,46$ & $44(113)$ & 89 & $80(100)$ \\
II & 94 & $110,4 \pm 20,01$ & $50(156)$ & 110 & $96,5(123)$ \\
III & 20 & $130,5 \pm 14,18$ & $107(164)$ & 126 & $121,5(141)$ \\
IV & 2 & $143,5 \pm 14,85$ & $133(154)$ & 143,5 & \\
\hline
\end{tabular}




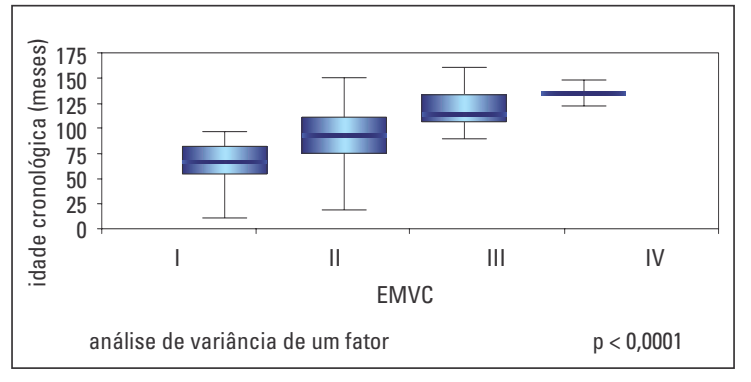

GRÁFICO 2 -Distribuição dos pacientes $(\mathrm{n}=140$ ) de acordo com o EMVC e a idade cronológica.

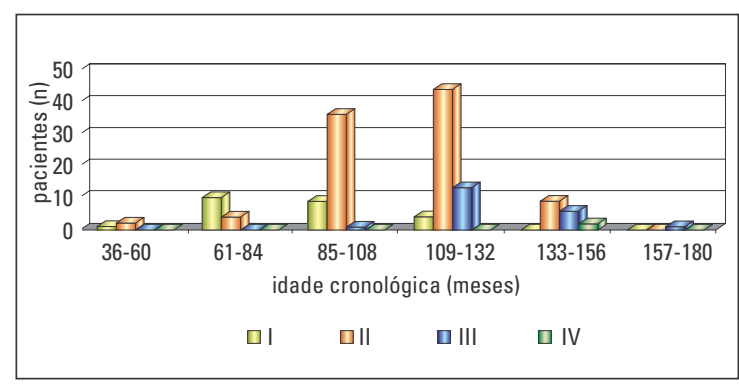

GRÁFICO 3 - Distribuição do número de pacientes $(n=140)$ por EMVC e idade cronológica.

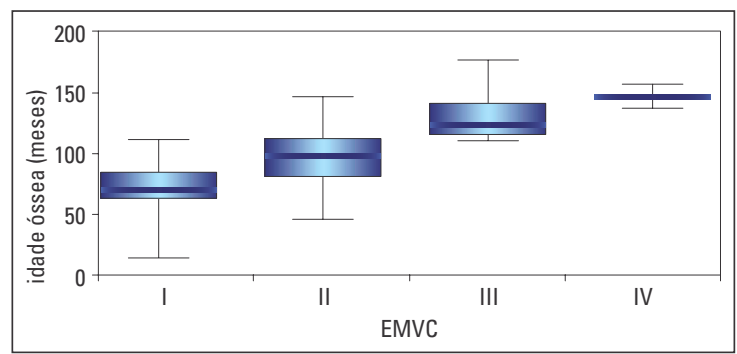

GRÁFICO 4 - Distribuição da idade óssea com os EMVC.

Variação dos estágios de maturação das vértebras cervicais C2, C3 e C4 e a idade óssea entre os gêneros masculino e feminino

Quando os pacientes foram separados em dois grupos (de acordo com o gênero), observou-se para a correlação entre os valores da idade óssea e os EMVC - os valores do coeficiente de correlação de Spearman, conforme a tabela 3.

Os valores demonstraram que o gênero feminino encontrou-se, tanto na idade óssea quanto nos EMVC, mais adiantado que o masculino, com
Tabela 2 - Coeficiente de correlação de Spearman entre os valores da idade cronológica e os EMVC para cada gênero.

\begin{tabular}{cccc}
\hline gênero & $\begin{array}{c}\text { coeficiente de } \\
\text { Spearman }(\mathbf{r})\end{array}$ & $\begin{array}{c}\text { intervalo de } \\
\text { confiança 95\% }\end{array}$ & p \\
\hline masculino & 0,5016 & 0,2887 a 0,6672 & $<0,0001$ \\
feminino & 0,6976 & 0,5532 a 0,8012 & $<0,0001$ \\
\hline
\end{tabular}

Tabela 3 - Coeficiente de correlação de Spearman entre os valores da idade óssea e os EMVC para cada gênero.

\begin{tabular}{cccc}
\hline gênero & $\begin{array}{c}\text { coeficiente de } \\
\text { Spearman }(\mathbf{r})\end{array}$ & $\begin{array}{c}\text { intervalo de } \\
\text { confiança 95\% }\end{array}$ & p \\
\hline masculino & 0,5071 & 0,2955 a 0,6713 & $<0,0001$ \\
feminino & 0,7312 & 0,5991 a 0,8246 & $<0,0001$ \\
\hline
\end{tabular}

uma correlação considerada positiva forte, portanto, altamente significante.

\section{DISCUSSÃO}

Os diferentes enfoques metodológicos aplicados para estimar a velocidade do crescimento puberal têm tentado explicar a sua correlação com o crescimento das estruturas craniofaciais, porque a identificação desta fase tem se mostrado uma boa referência para a previsão da época de maior velocidade de crescimento facial, favorecendo o plano de tratamento ortodôntico/ortopédico. Sua determinação pode resultar da utilização de vários métodos, simultânea ou separadamente, como a idade estatural; a idade óssea pela maturação dos ossos da mão e punho e das vértebras cervicais; a idade morfológica; a idade sexual e a idade dentária ${ }^{9,16,22,30}$.

Entre os vários indicadores de crescimento, a idade esqueletal talvez seja a mais precisa, seguida pelas características sexuais secundárias, pelos aspectos morfológicos e pela idade cronológica $8,12,13,14,16,18,19,28,29,30$.

Os métodos usados para a avaliação da idade óssea pelos eventos de maturação dos ossos da mão e punho são comumente divididos em dois tipos principais: o clássico, também denominado 
tipo padrão-etário ou inspecional, que consiste na comparação da radiografia obtida com as radiografias de um Atlas, como o de Greulich e Pyle ${ }^{15}$; o método de escores, quando cada centro de ossificação recebe um número de pontos de acordo com uma escala de desenvolvimento, como no sistema de Tanner e Whitehouse; métodos que utilizam medidas dimensionais dos centros de ossificação na avaliação, como o índice de Schmid e Moll e o método de Eklof e Ringertz ${ }^{4,5,12,28,29,30}$.

No campo da Ortodontia, a grande maioria dos estudos que pesquisam a idade biológica visando estabelecer o surto de crescimento puberal tem utilizado o método de maturação das vértebras cervicais proposto por Lamparski ${ }^{17}$, modificado por Hassel e Farman ${ }^{16}$, buscando determinar sua correlação com os eventos de maturação dos ossos da mão e punho. Este método tem sido considerado um bom indicador biológico nos planejamentos ortodônticos, por ser direto e de observação simples, sem necessidade de exposição a radiação adicional, por ser realizado em telerradiografias laterais cranianas que, rotineiramente, já fazem parte do protocolo da documentação ortodônti-

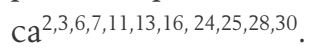

O método utilizado nesta pesquisa foi o de Hassel e Farman ${ }^{16}$ modificado por Baccetti, Franchi e McNamara Jr. ${ }^{2}$ Este, que apresenta 5 EMVC e tem correlação com o crescimento puberal, foi comparado com a maturação dos ossos da mão e punho pelo método de Greulich e Pyle ${ }^{15}$.

Quando analisada a correlação da idade cronológica com a maturação das vértebras cervicais ficou claro que - com o aumento da idade cronológica - ocorreu um avanço proporcional dos EMVC. Esta proporcionalidade pôde ser encontrada no gráfico 3 , quando avaliada a distribuição dos pacientes por faixa etária, com maior prevalência de pacientes na faixa entre 85 e 108 meses ( 7 a 9 anos) e entre 109 e 132 meses ( 9 a 11 anos), correspondentes ao estágio II da MVC, considerado o melhor momento para iniciar os tratamentos ortodônticos/ortopédicos ${ }^{2,3,13}$.
Os resultados desta pesquisa demonstraram que houve correlação positiva, porém estatisticamente significante, dos EMVC com a idade cronológica, com $r=0,5830$ e $p<0,0001$ (Gráf. 2). Esta mesma correlação foi encontrada em outros estudos que demonstraram, pelos indicadores de MVC, que os pacientes encontravam-se também no surto de crescimento puberal ${ }^{11,13,16,21}$.

Quando associados aos eventos de ossificação da mão e punho no momento do pico de crescimento (Gráf. 4), o período de crescimento prépuberal correspondeu ao estágio I das MVC; o pico de crescimento puberal correspondeu aos estágios II e III, e o período pós-puberal, aos estágios IV e $\mathrm{V}^{1,2,3,6,10,11}$.

Constatou-se uma correlação positiva moderada dos EMVC com a maturação dos ossos da mão e punho, com $\mathrm{r}=0,6326$ e $\mathrm{p}<0,0001$ (Gráf. 4). Com estes dados, constatou-se que a maior prevalência dos pacientes encontrou-se no estágio II de MVC, correspondente a 108 meses da idade óssea (9 anos), confirmada em outros estudos ${ }^{1,10,13}$.

Quando os pacientes foram separados de acordo com o gênero e correlacionados com os EMVC, os resultados demonstraram que o gênero feminino encontrou-se mais adiantado que o masculino, com uma correlação considerada entre positiva média e positiva forte (Tab. 3). O mesmo foi encontrado em outras pesquisas, porque sabe-se que o gênero feminino atinge sua forma facial adulta numa idade consideravelmente mais jovem, sendo que a idade cronológica média das meninas, no pico do surto de crescimento puberal, é significantemente inferior à dos meninos 5,6,8,13,14,30.

Há uma tendência do gênero feminino alcançar o pico de crescimento puberal um ano antes do masculino ${ }^{6}$, como demonstrado pelos resultados deste estudo (Tab. 2), onde as meninas atingiram o pico de crescimento, em média, entre 106 e 131 meses, e os meninos entre 120 e 137 meses.

Para Schusterchitz e Haiter Neto ${ }^{26}$, de um modo geral na população brasileira, o pico de crescimento puberal ocorre mais precocemente, 
quando comparado com dados descritos na literatura mundial.

Não pode deixar de ser enfatizado que a avaliação do crescimento puberal por meio das alterações morfológicas da MVC é um método adicional, que deve ser complementado pelas radiografias de mão e punho e por todas as informações que permitam estabelecer um diagnóstico e um planejamento mais seguros.

\section{CONCLUSÕES}

Após a análise dos resultados obtidos, julga-se válido concluir que:

1) $\mathrm{Na}$ correlação das alterações das vértebras cervicais C2, C3 e C4 com a idade cronológica, verificou-se que a idade aumenta proporcionalmente em relação aos estágios de maturação das vértebras cervicais, sendo que a maioria dos pa- cientes da amostra encontrou-se no estágio II, considerado o melhor momento para o início da terapêutica ortodôntico/ortopédica. Na comparação da idade esqueletal com os ossos da mão e punho, o nível de correlação entre os dois métodos demonstrou que a avaliação dos estágios de maturação das vértebras cervicais é um método adicional útil e confiável na determinação do estágio de crescimento facial nas crianças em crescimento puberal.

2) O gênero feminino apresentou estágios de crescimento antes do gênero masculino, atingindo o início e o final do surto puberal em idade cronológica inferior.

Cervical vertebral maturation and the correlation between the skeletal age of the hand-wrist as orthodontic treatment indicators

\begin{abstract}
Aim: The aim of this prospective, transversal, multicentric study was to determine the correlation between the morphologic changes of the second, third and fourth cervical vertebral bodies and the chronological age; to compare with the skeletal age of the hand and wrist bones; and to verify the differences in the bone growth pattern between male and female. Methods: The sample was constituted by 140 subjects, 74 girls and 66 boys. The morphologic alterations of the cervical vertebrae was analyzed by the Hassel and Farman method modified by Baccetti, Franchi and McNamara to 5 stages that correlate to the puberal growth in lateral cephalometric radiographs. The handwrist radiographs evaluated the events of ossification by the method of Greulich and Pyle. Results: The results demonstrated: correlation of the stages of cervical vertebral maturation with the chronological age; positive and moderate significant correlation between the two methods with $r=0.6326(I C=95 \%)$ and $p<0.0001$. Conclusion: It was concluded that the age increases proportionally in relation to the cervical vertebral maturation stages, where the majority of the patients of the sample in the stage II were considered good for the beginning of the orthodontic and orthopedic therapy. The comparison between the two methods demonstrated that the cervical vertebral maturation stages is a useful and trustworthy additional method in the determination of children's facial and puberal growth. Females presented earlier growth stages than males, reaching the beginning and end of pubertal growth spurt in earlier age.
\end{abstract}

Key words: Skeletal maturation. Puberal growth. Hand-wrist maturation. Cervical vertebrae. 


\section{REFERÊNCIAS}

1. BACCETTI, T. et al. Treatment timing for Twin-block therapy. Am. J. Orthod. Dentofacial Orthop., St. Louis, v. 118, no. 2, p. 159-170, 2000

2. BACCETTI, T.; FRANCHI, L.; McNAMARA JR., J. A. An improved version of cervical vertebral maturation (CVM) method for the assessment of mandibular growth. Angle Orthod. Appleton, v. 72, no. 4, p. 316-323, 2002

3. BACCETTI, T.; FRANCHI, L.; MCNAMARA, JR., J. A. The cervical vertebral maturation method: some need for clarification. Am. J. Orthod. Dentofacial Orthop., St. Louis, v. 123, no. 1, p. 19A-20A, Jan. 2003.

4. BENEMANN, E.; SAMPAIO, R.; BERTHOLD, T. Idade biológica: indicadores do surto de crescimento. Ortodon. Gauch. Porto Alegre, v. 1, n. 1, p. 62-76, abr. 1997

5. BYDLOWSKI, S. P. Fisiologia da determinação e diferenciação sexual. In: DOUGLAS, C. R. Tratado de Fisiologia aplicado às ciências da saúde. São Paulo: Robe Editorial, 1994. p. $1123-1132$

6. CANALI, L.; BRÜCKER, M. R.; LIMA, E. M. S. Avaliação da maturação esquelética das vértebras cervicais através de telerradiografias de perfil. Rev. Odonto Cienc., Porto Alegre, v. 18, n. 40, p. $127-137,2003$.

7. CHANG, H. P. et al. Correlation of cervical vertebrae maturation with hand-wrist maturation in children. Kaohsiung J. Med. Sci., Kaohsiung, v. 17, no. 1, p. 29-35, 2001

8. DEMIRJIAN, A. et al. Interrelations among measures of somatic, skeletal, dental, and sexual maturity. Am. J. Orthod., St. Louis, v. 88, no. 5, p. 433-438, 1985

9. FALTIN JR., K. Ortodontia funcional dos maxilares. In: PETRELLI, E. Ortodontia contemporânea. São Paulo: Sarvier, 1988. p. $211-246$

10. FALTIN JR, K. et al. Long-term effectiveness and treatment timing for bionator therapy. Angle Orthod., Appleton, v. 73, no. 3, p. 221-230, 2003

11. FRANCHI, L.; BACCETTI, T.; McNAMARA JR., J. A. Mandibular growth as related to cervical vertebral maturation and body height. Am. J. Orthod. Dentofacial Orthop., St. Louis, v. 118 , no. 3, p. 335-340, Sept. 2000.

12. FISHMAN, L. S. Radiographic evaluation of skeletal maturation: a clinically oriented method based on hand-wrist films. Angle Orthod., Appleton, v. 52, no. 2, p. 88-112, Apr. 1982.

13. GEORGEVICH JR, R. Correlações entre a maturação dos ossos da região metacarpofalângica do polegar, estágios de desenvolvimento dos dentes caninos e segundos molares permanentes e alterações morfológicas das vértebras cervicais. Trabalho de Conclusão de Curso (Especialização)Faculdade de Odontologia, Universidade Paulista, São Paulo, 2000.

14. GENEROSO, R. et al. Estudo da correlação entre a idade cronológica e a maturação das vértebras cervicais em pacientes em fase de crescimento puberal. Rev. Dental Press Ortodon. Ortop. Facial, Maringá, v. 8, n. 4, p. 19-36, jul./ago. 2003
15. GREULICH, W. W.; PYLE, S. I. Radiographic atlas of skeletal development of hand and wrist. 2nd ed. Califórnia: Stanford University, 1959

16. HASSEL, B.; FARMAN, A. G. Skeletal maturation evaluation using cervical vertebrae. Am. J. Orthod. Dentofacial Orthop., St. Louis, v. 107, no. 1, p. 58-66, Jan. 1995.

17. LAMPARSKI, D. C. Skeletal age assessment utilizing cervical vertebrae.1972. Thesis (Master of Dental Science)-University of Pittsburgh, Pittsburgh, 1972

18. LANGLADE, M. Céphalométrie orthodontique. Paris: Maloine, 1978.

19. MARTINS, R. J. C.; SAKIMA, T. Considerações sobre a previsão do surto de crescimento puberal. Ortodontia, São Paulo, v. 10, n. 3, p. 164-170, set./dez.1977

20. MEDICUS, H.; GRÖNA, A. M.; MOORREES, C. F. A. Reproducibility of rating stages of osseous development (Tanner-Whitehouse system). Am. J. Phys. Anthropol., New York, no. 35, p. 359-372, 1971.

21. MITO, T.: SATO, K.: MITANI, H. Cervical vertebral bone age in girls. Am. J. Orthod. Dentofacial Orthop., St. Louis, v. 122, no. 4, p. 380-385, Oct. 2002.

22. RICKETTS, R. M. The influence of orthodontic treatment on facial growth and development. Angle Orthod., Appleton, v. 30 , no. 3, p. 103-133, 1960.

23. RICKETTS, R. M. Orthodontic diagnosis and planning Denver: Rocky Mountain Data Systems, 1982.

24. SÁ FILHO, F. P. G. As bases fisiológicas da Ortopedia Maxilar. São Paulo: Ed. Santos, 1999.

25. SANTOS, S. C. B. N.; ALMEIDA, R. R. Estudo comparativo de dois métodos de avaliação esquelética utilizando telerradiografias em norma lateral e radiografias carpais. Ortodontia, São Paulo, v. 32, n. 2, p. 33-45, maio/ago. 1999.

26. SCHUSTERCHITZ, T.; HAITER NETO, F. Estudo comparativo entre a maturação das vértebras cervicais e a região carpal. Ortodontia, São Paulo, v. 35, n. 3, p. 33-42, jul./set. 2002.

27. TANNER, J. M. et al. Assessment of skeletal maturity and prediction of adult height (TW2 method). 2nd ed. London: Academic Press, 1983.

28. TAVANO, O. Estudo das principais tabelas de avaliação da idade biológica, através do desenvolvimento ósseo, visando sua aplicação em brasileiros leucodermas da região de Bauru. Tese (Doutorado)-Faculdade de Odontologia de Bauru, Universidade de São Paulo, São Paulo, 1976.

29. VAN DER LINDEN, F. P. G. De morfologie van de cranio-faciale strukturen in verband met de extra-orale therapie. Rev. Belge Méd. Dent., Amsterdam, v. 29, no. 2, p. 265-268, 1974.

30. VAN DER LINDEN, F. P. G. Crescimento e Ortopedia Facial. 2. ed. Rio de Janeiro: Quintessence, 1990.
Endereço para correspondência

Vitoria Aparecida Muglia Moscatiello

Av. dos Bandeirantes, 3426 - Campo Belo

CEP: 04.071-000 - São Paulo / SP

E-mail: moscatiello@apcd.org.br 